\title{
Périodes d'activités et structure sociale de Cephalophus zebra Gray, 1838 et de Cephalophus jentinki Thomas, 1892 dans le Parc National de Taï, Sud-Ouest de la Côte d'Ivoire
}

\author{
Ange Edgar Habib MONKET ${ }^{1 *}$, Célestin Yao KOUAKOU ${ }^{1,2}$, Malé Roger KELY ${ }^{1,2}$, \\ André Djaha KOFFI ${ }^{1,2}$, Antoine Kouamé N'GUESSAN ${ }^{1}$, Emmanuelle NORMAND ${ }^{3}$, \\ Yves Aka KABLAN ${ }^{3,4}$, Abdoulaye DIARRASSOUBA ${ }^{5}$, Adama TONDOSSAMA ${ }^{5}$ et \\ Jean-Claude Koffi BENE ${ }^{1}$
}
${ }^{1}$ Laboratoire de Biodiversité et Ecologie Tropicale de l'UFR Environnement de l'Université Jean Lorougnon Guédé, Daloa, Côte d'Ivoire, BP 150 Daloa, Côte d'Ivoire.
${ }^{2}$ Centre Suisse de Recherches Scientifiques en Côte d'Ivoire, 01 BP 1303 Abidjan 01, Côte d'Ivoire.
${ }^{3}$ Wild Chimpanzee Foundation, 23 BP 238 Abidjan 23, Côte d'Ivoire.
${ }^{4}$ UFR Biosciences, Université Felix Houphouët-Boigny, 22 BP 582 Abidjan 22, Côte d'Ivoire.
${ }^{5}$ Office Ivoirien des Parcs et Réserves, 06 BP 426 Abidjan 06, Côte d'Ivoire.
*Auteur correspondant, E-mail : angeedgarmonket@gmail.com; Tel : +2250758231814
Received: 17-07-2021
Accepted: 05-10-2021
Published: 30-10-2021

\section{RESUME}

La connaissance du profil écologique de la faune d'un site est importante pour mieux apprécier la dynamique spatio-temporelle des espèces et les interactions entre elles. La présente étude a été réalisée au Parc National de Taï. Elle a pour objectif de déterminer les périodes d'activités nycthémérales et la structure sociale du céphalophe zébré et du céphalophe de Jentink. Au total, 291 pièges photographiques ont été installés suivant un dispositif systématique de mars 2019 à mars 2020. Le traitement des enregistrements vidéo obtenus a permis de montrer que le céphalophe zébré est préférentiellement diurne avec $84 \%$ des observations faites dans la journée. Les périodes d'activités maximales de cette espèce ont été marquées en début de journée de 6 à $8 \mathrm{~h}$ et en fin d'après-midi de 15 à $17 \mathrm{~h}$. Par contre, le céphalophe de Jentink est principalement nocturne avec $83 \%$ des observations enregistrées la nuit. Cette espèce a été plus active autour de $2 \mathrm{~h}, 4 \mathrm{~h}$ et $19 \mathrm{~h}$. Cependant, ces deux espèces mènent une vie généralement solitaire avec parfois une formation de groupes de deux individus adultes. Pour le céphalophe zébré, 94,21\% des individus vivent seul. Concernant le céphalophe de Jentink, 96,70\% des individus solitaires ont été identifiés.

(C) 2021 International Formulae Group. All rights reserved.

Mots clés : céphalophe zébré, céphalophe de Jentink, Parc National de Taï, périodes d'activités, structure sociale.

\section{Activity periods and social structure of Zebra duiker Gray, 1838 and Jentink's duiker Thomas, 1892 in Taï National Park, southwestern Côte d'Ivoire}

\begin{abstract}
The knowledge of the ecological profile of the fauna of a site is important to better appreciate the spatio-temporal dynamics of species and the interactions between them. The present study was carried out in the Taï National Park. Its objective is to determine the periods of nycthemeral activities and the social structure of
\end{abstract}


zebra duiker and Jentink's duiker. A total, of 291 camera traps were set in a systemic design from March 2019 to March 2020. The processing of the obtained video recordings showed that the zebra duiker is preferentially diurnal with $84 \%$ of the observations made during the day. The periods of maximum activity of this species were marked at the beginning of the day from 6 to 8 am and in the late afternoon from 3 to $5 \mathrm{pm}$. In contrast, Jentink's duiker is mainly nocturnal with $83 \%$ of observations recorded at night. This species was most active around $2 \mathrm{~h}$, $4 \mathrm{~h}$ and $19 \mathrm{~h}$. However, these two species lead a generally solitary life with sometimes a groups formation of two adult individuals. For the zebra duiker, $94.21 \%$ of individuals live alone. Concerning the Jentink's duiker, $96.70 \%$ of the solitary individuals have been identified.

(C) 2021 International Formulae Group. All rights reserved.

Keywords: Zebra duiker, Jentink’s duiker, Taï National Park, activity periods, social structure.

\section{INTRODUCTION}

Le Sud-Ouest ivoirien constitue l'une des régions prioritaires pour la conservation de l'écosystème forestier de la Haute Guinée (Lauginie, 2007). C'est en raison de sa diversité biologique et des endémismes qui la caractérisent que cette région a été identifiée comme l'un des plus importants «hotspots» pour la biodiversité mondiale (Myers et al., 2000 ; Kuper et al., 2004 ; Mittermeier et al., 2011). Malgré cette diversité biologique exceptionnelle, ce bloc forestier subit une forte pression anthropique consécutive à l'agriculture, au braconnage et à l'orpaillage artisanal clandestin. Parmi ces menaces humaines précitées, l'agriculture est l'une des pires formes de destruction des forêts du SudOuest ivoirien (Yéo et al., 2011 ; Koné et al., 2014).

Le Parc National de Taï (PNT) qui appartenant à cette région, est un site privilégié pour l'étude des ongulés de forêt. En effet, sur les 6 familles de ces mammifères qui sont représentés en Afrique, ce site renferme 5 familles (Elephantidae, Hippopotamidae, Suidae, Tragulidae et Bovidae) réparties en 10 genres et 15 espèces (Lauginie, 2007). Parmi ces ongulés, deux espèces de la famille des bovidés sont menacées d'extinction et sont endémiques pour la région de l'ouest de la Sierra Léone jusqu'au Sud-Ouest de la Côte d'Ivoire. Il s'agit du céphalophe zébré (Cephalophus zebra Gray, 1838) et du céphalophe de Jentink (Cephalophus jentinki Thomas, 1892) (Diarrassouba et al., 2019).
Vu la dégradation des massifs forestiers à l'Ouest de la Côte d'Ivoire, le Parc National de Taï serait le dernier refuge pour ces espèces animales (Lauginie, 2007). Malheureusement, à ce jour, très peu d'études ont concerné particulièrement ces deux espèces de céphalophes (Tiedoué et al., 2018). En effet, une étude des adaptations des céphalophes aux perturbations de l'environnement débutée en 1989 dans le parc, n'a pu être menée jusqu'à terme (Lauginie, 2007). Au-delà de cette étude, celles qui ont pu être menées dans le parc, ont porté généralement sur toutes les 7 espèces de céphalophes (Cephalophus dorsalis, Philantomba maxwelli, Cephalophus niger, Cephalophus sylvicultor, Cephalophus jentinki, Cephalophus zebra et Cephalophus ogilbyi (Lauginie, 2007 ; Diarrassouba et al., 2019). Elles ont traité notamment, de leur abondance et de leur distribution (Tiedoué et al., 2015, 2016, 2018), l'effet des facteurs naturels et anthropiques sur celles-ci (Diarrassouba et al., 2019) et leurs périodes d'activités (N'Goran et al., 2020). De plus, l'étude concernant les périodes d'activités des céphalophes n'a été menée que dans la zone de recherche en écologie du PNT. Par conséquent, une étude couvrant toute l'étendue du parc, permettant à apporter des connaissances sur les périodes d'activités et la structure sociale de ces deux espèces de céphalophes, menacées d'extinction et endémiques au PNT, s'avère nécessaire.

Cette étude vise donc à renseigner les périodes d'activités et la structure sociale du 
céphalophe zébré (Cephalophus zebra) et du céphalophe de Jentink (Cephalophus jentinki).

\section{MATERIEL ET METHODES}

\section{Site d'étude}

Cette étude a été réalisée dans le Parc National de Taï situé entre $5^{\circ} 10^{\prime}$ et $6^{\circ} 50^{\prime}$ de latitude nord et entre $6^{\circ} 50^{\prime}$ et $7^{\circ} 50^{\prime} \mathrm{de}$ longitude ouest (Figure 1). C'est le plus grand parc national sous statut strict de protection en Afrique de l'Ouest. Il couvre avec la Réserve partielle de Faune du N'Zo, une superficie de 536016 hectares (Diarrassouba et al., 2019). Il est caractérisé par la présence d'une végétation dense humide de type «sempervirente ou forêt ombrophile ». $\mathrm{Au}$ regard de sa valeur inestimable du point de vue de sa diversité floristique et faunique et des services écosystémiques qu'il fournit, le Parc national de Taï a été classé en "réserve de biosphère » en 1978, puis inscrit sur la liste du patrimoine mondial de l'UNESCO en 1982 (Lauginie, 2007). La moyenne des précipitations annuelles est de $1800 \mathrm{~mm}$ et la température moyenne annuelle est de $25^{\circ} \mathrm{C}$ (Brou, 2009).

\section{Collecte des données}

La méthode des transects en point adaptée aux pièges photographiques (Howe et al., 2017) a été utilisée pour la collecte des données de terrain. Cette étude a été conduite de mars 2019 à mars 2020. Un dispositif systématique d'échantillonnage a été mis en place en constituant une grille de maille $4 \times 4$ $\mathrm{km}$ qui a été superposé sur la carte du parc, à l'aide du logiciel Qgis 2.18.7. Chaque point projeté pour constituer cette grille a été pris comme le point d'installation d'un piège photographique (PP) (modèle Bushnell, Trophy Cam HD Aggressor). En somme, 4 pièges photographiques au maximum étaient posés par grille et ils étaient distants de $4 \mathrm{Km}$ les uns aux autres sur toute l'étendue du parc. Le dispositif final se composait de 291 points d'installation de PP (Figure 1). Pour l'échantillonnage de ces 291 points, $200 \mathrm{PP}$ ont été utilisés. A chacun de ces points, en forêt, un PP était orienté, à $0^{\circ}$ ou $180^{\circ}$ (direction NordSud) pour éviter les rayons solaires dans l'objectif des pièges photographiques, avec souvent une déviation $\left( \pm 20^{\circ}\right)$ pour pallier les problèmes d'observation dus à certains obstacles (chablis, cours d'eau, végétation très dense). Chaque PP a été installé dans un rayon de $30 \mathrm{~m}$ des points théoriques préenregistrés dans un GPS (Global Positioning System) en privilégiant un «meilleur arbre ». Au final, le piège était fixé sur un arbre d'environ $10 \mathrm{~cm}$ de diamètre, à une hauteur de $50 \mathrm{~cm}$ au-dessus du sol. Les paramètres définis pour les PP étaient le mode vidéo, une sensibilité élevée au mouvement, un enregistrement de 60 secondes (s) à la suite de déclenchement par mouvement, un intervalle minimum de $2 \mathrm{~s}$ entre deux enregistrements ainsi qu'une inscription de la date et l'heure d'enregistrement de chaque vidéo. Les PP ont été retirés deux mois plus tard à compter de la date de pose, puis placés ailleurs. Chaque point d'installation de PP a été piégé qu'une seule fois pendant la période d'échantillonnage. Aucune autre visite était effectuée pour le renouvellement des bactéries.

Au total, 267 sites ont été correctement échantillonnés. La somme des différents efforts d'échantillonnage des PP a ainsi donné un effort d'échantillonnage global de 169646 jours.

La reconnaissance de ces espèces de céphalophes a été possible grâce à une formation de reconnaissance initiée par la WCF (Wild Chimpanzee Foundation) en collaboration avec l'OIPR (Office Ivoirien des Parcs et Réserves) de la faune du Parc National de Taï. En plus de cette formation, nous avons utilisé également le guide de reconnaissance des mammifères d'Afrique (Kingdon et al., 2013).

\section{Analyse des données \\ Détermination des périodes d'activités et de la structure sociale}

Les vidéos recueillies des PP ont été stockées sur un disque dur, puis visualisées à l'ordinateur à l'aide d'un logiciel de lecture de vidéo (VCL media player 3.0.2 64-bit). En vue d'effectuer des analyses, les données ont été enregistrées dans une feuille Excel.

Pour une position de PP donnée, les détections successives d'individus de la même 
espèce, dans un laps de temps inférieur à 30 minutes, ont été considérées comme un seul évènement ou visite (Bezerra et al., 2014 ; Green et al., 2018 ; Hedwig et al., 2018 ; Kely, 2020). Cependant, pour la même position, une détection qui intervient après un intervalle de temps supérieur ou égal à 30 minutes de la première détection de la visite précédente, est considérée comme indépendante et marque le début d'une nouvelle visite (Bezerra et al., 2014 ; Green et al., 2018). L'évènement ou la visite représente un ensemble d'enregistrements successifs (vidéos) intervenant dans un laps de temps inférieur à 30 minutes. Ces enregistrements sont supposés présenter le même individu qui se traine dans le champ de vision du PP ou les membres d'un groupe de céphalophes (Ancrenaz et al., 2012 ; Wearn et Glover-Kapfer, 2017).

Pour les périodes d'activités nycthémérales, l'heure de début du premier enregistrement vidéo de chaque évènement de capture de chaque individu de céphalophe a été considérée comme l'heure de capture de l'évènement (Howe et al., 2017 ; Gaynor et al., 2018 ; Green et al., 2018 ; Kely, 2020). Les heures de capture des différents évènements des deux espèces de céphalophes ont permis de déterminer leurs périodes d'activités nycthémérales, en suivant la classification de Gómez et al. (2005) indiqué dans le Tableau 1. En Côte d'Ivoire, le lever du soleil intervient aux alentours de $6 \mathrm{~h} 00$ et le coucher du soleil aux alentours de $18 \mathrm{~h} \mathrm{00,} \mathrm{heure} \mathrm{locale} \mathrm{(UTC} \mathrm{+}$ $0)$. A cet effet, l'activité diurne a été classée comme celle ayant lieu entre 6 h 30 min et 17 h $30 \mathrm{~min}$ et l'activité nocturne entre 18 h 30 min et $5 \mathrm{~h} 30 \mathrm{~min}$; les périodes restantes (entre 5 h 30 et 6 h $30 ; 17$ h 30 et 18 h 30) ont été dites crépusculaires (Ross et al., 2013).

Quant à la structure sociale, après le regroupement des détections en évènements, nous avons d'abord décompté le nombre d'individus distincts pour chaque évènement. Ce nombre est considéré comme la taille du groupe observé (Bezerra et al., 2014 ; Hedwig et al., 2018 ; McCarthy et al., 2018 ; Kely, 2020). Nous avons ensuite, calculé la taille moyenne des groupes de chaque espèce de céphalophe avec les individus solitaires et la taille moyenne des groupes sans les individus solitaires. Enfin, nous avons déterminé la classe d'âge des individus à partir des traits morphologiques tels que la taille relative des individus et l'absence ou la taille des cornes (Newing, 2001 ; Kely, 2020).

Tableau 1 : Périodes d'activités selon la classification de Gómez et al. (2005).

\begin{tabular}{ll}
\hline Périodes d'activités & Définition \\
\hline Espèces diurnes & Moins de $10 \%$ des observations dans la nuit \\
\hline Espèces plus diurnes que nocturnes & 10 à $30 \%$ des observations dans la nuit \\
\hline Espèces cathémérales & 30 à $70 \%$ des observations dans la nuit \\
\hline Espèces plus nocturnes que diurnes & 70 à $90 \%$ des observations dans la nuit \\
\hline Espèces nocturnes & Plus de $90 \%$ des observations dans la nuit \\
\hline Espèces crépusculaires & $50 \%$ des observations au crépuscule \\
\hline
\end{tabular}




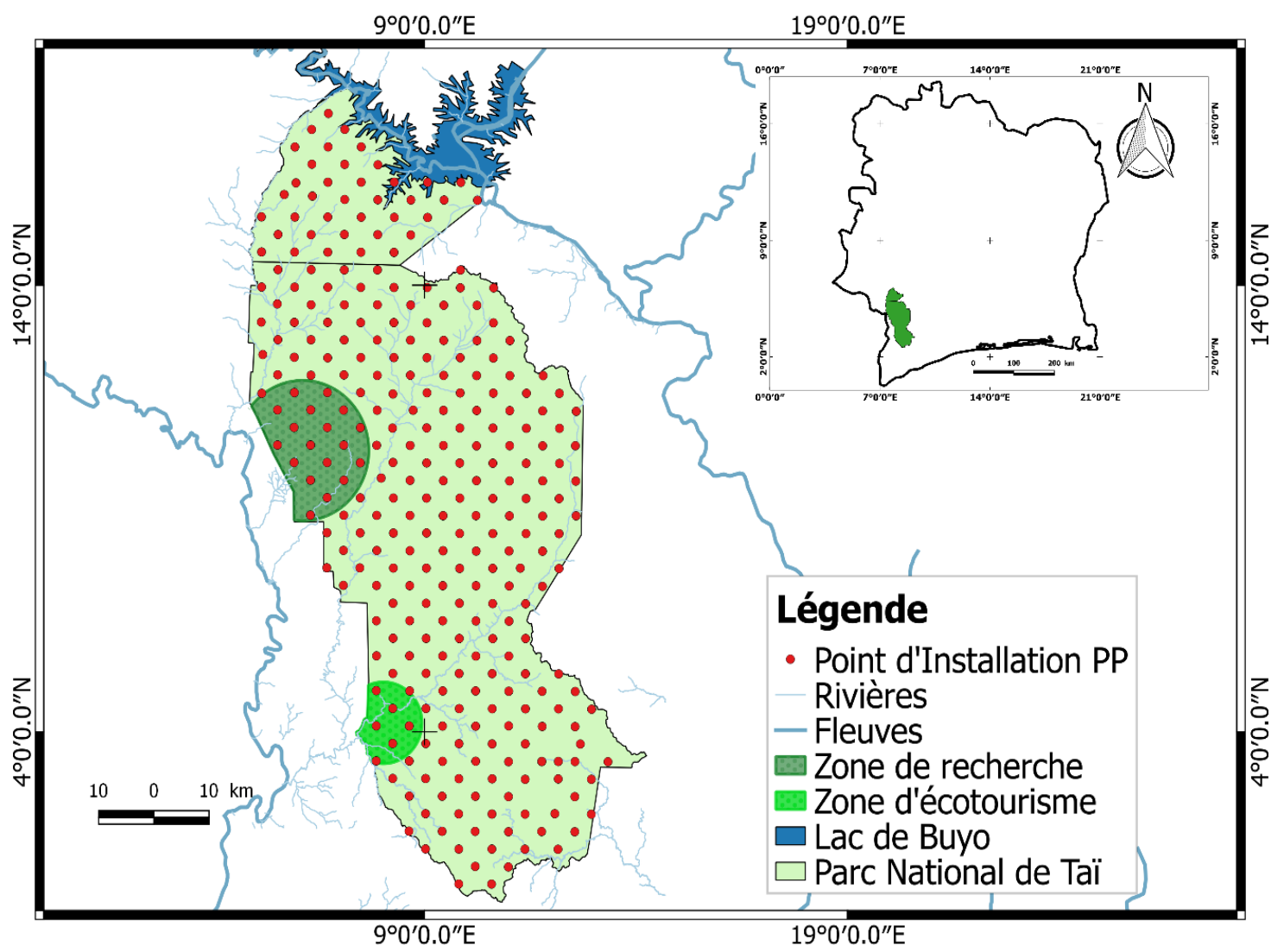

Figure 1 : Localisation du Parc National de Taï et le dispositif des pièges photographiques.

\section{RESULTATS}

Au terme de la période de piégeage, les données de 267 PP ont été prises en compte dans notre analyse. Ces 267 PP ont enregistré 1366 vidéos dont 760 évènements de capture (vidéos) du céphalophe zébré et 606 enregistrements de capture du céphalophe de Jentink.

\section{Périodes d'activités}

Le céphalophe zébré est principalement actif pendant la journée. Cette activité diurne est nettement bimodale avec un maximum de déplacements au début de la journée (de $6 \mathrm{~h} 30$ $\min$ à $7 \mathrm{~h} 30 \mathrm{~min}$ ) et un autre vers la fin d'aprèsmidi (de 15 à 17h). Ainsi, une analyse du nombre d'évènements de capture pendant chaque période du nycthémère, révèle que sur les 760 évènements de capture de cette espèce,
$634(84 \%)$ ont été faits la journée, contre 71 (9\%) au crépuscule et 55 (7\%) la nuit. Nous constatons ainsi que le céphalophe zébré est une espèce diurne au Parc National de Taï (Figure 2). A l'opposé, le céphalophe de Jentink est majoritairement actif la nuit. Cette mobilité nocturne est caractérisée par trois maxima de déplacements, de $1 \mathrm{~h} 30 \mathrm{~min}$ à $2 \mathrm{~h} 30$ min, de $3 \mathrm{~h} 30$ min à $5 \mathrm{~h}$ et de $18 \mathrm{~h} 30$ min à $19 \mathrm{~h} 30$ min. A cet effet, une analyse du nombre d'évènements de capture pendant chaque période du nycthémère, révèle que sur les 606 évènements de capture, 502 évènements de capture ont été enregistrés la nuit, soit 83\%, 81 évènements de capture au crépuscule, soit $13 \%$ et 23 évènements de capture dans la journée, soit $4 \%$. Au regard de ce constat, nous pouvons noter que le céphalophe de Jentink est plus nocturne que diurne (Figure 2). La Figure 3 
illustre successivement des photographies d'un céphalophe zébré et d'un céphalophe de Jentink.

\section{Structure sociale}

Pour le céphalophe zébré, la taille moyenne des groupes y compris les individus solitaires est de $1 \pm 0,27$ individu. Les groupes ( 2 individus) ont été observés à 44 reprises correspondant à $5,79 \%$ des évènements $(\mathrm{N}=$ 760) contre 716 évènements des individus solitaires soit $94,21 \% \quad(\mathrm{~N}=760)$ des évènements. On peut donc dire que le céphalophe zébré au Parc National de Taï, vit préférentiellement en solitaire. La taille maximale de groupe a été de 2 individus. Quant à la répartition des individus en classes d'âge, il a été dénombré uniquement que 760 adultes, représentant $100 \%$ de la population (Tableau 2).
Concernant le céphalophe de Jentink, la taille moyenne de groupe est de $1 \pm 0,33$ individu, avec les individus solitaires. Les groupes ( 2 individus) ont été identifiés 20 fois représentant $3,30 \%$ des évènements $(\mathrm{N}=606)$. Par contre, 586 évènements des individus solitaires ont été observés soit $96,70 \%(\mathrm{~N}=$ 606). Au regard de ces chiffres, nous pouvons avancer que le céphalophe de Jentink est principalement solitaire au Parc National de Taï. La taille maximale de groupe a également été de 2 individus. Au niveau de la répartition des individus par classes d'âge, nous n'avions dénombré essentiellement que 606 adultes soit $100 \%$ de la population (Tableau 3). La Figure 4 montre successivement un couple de céphalophe zébré et un autre de céphalophe de Jentink au Parc national de Taï.

Tableau 2 : Fréquence d'observation de céphalophe zébré au Parc National de Taï.

\begin{tabular}{lccccc}
\cline { 2 - 4 } & $\begin{array}{l}\text { Taille du } \\
\text { groupe }\end{array}$ & $\begin{array}{l}\text { Structure du } \\
\text { groupe }\end{array}$ & $\begin{array}{l}\text { Evènements } \\
\text { de capture }\end{array}$ & $\begin{array}{l}\text { Pourcentage } \\
\text { d'observation }\end{array}$ & $\begin{array}{l}\text { Moyenne } \\
\text { d'individus }\end{array}$ \\
\hline $\begin{array}{l}\text { Individus } \\
\text { solitaires }\end{array}$ & 1 & 1 Ad & 716 & 94,21 & 1 \\
Groupes & 2 & $2 \mathrm{Ad}$ & 44 & 5,79 & 2 \\
\hline & Total & & $\mathbf{7 6 0}$ & $\mathbf{1 0 0}$ \\
\hline Ad: Adulte & & & &
\end{tabular}

Tableau 3: Fréquence d'observation de céphalophe de Jentink au Parc National de Taï.

\begin{tabular}{lccccc}
\cline { 2 - 5 } & $\begin{array}{c}\text { Taille du } \\
\text { groupe }\end{array}$ & $\begin{array}{c}\text { Structure du } \\
\text { groupe }\end{array}$ & $\begin{array}{c}\text { Evènements } \\
\text { de capture }\end{array}$ & $\begin{array}{c}\text { Pourcentage } \\
\text { d'observation }\end{array}$ & $\begin{array}{c}\text { Moyenne } \\
\text { d'individus }\end{array}$ \\
\hline $\begin{array}{l}\text { Individus } \\
\text { solitaires }\end{array}$ & 1 & $1 \mathrm{Ad}$ & 586 & 96,7 & 1 \\
Groupes & 2 & $2 \mathrm{Ad}$ & 20 & 3,3 & 2 \\
\hline \multicolumn{1}{r}{} & Total & & $\mathbf{6 0 6}$ & $\mathbf{1 0 0}$ &
\end{tabular}




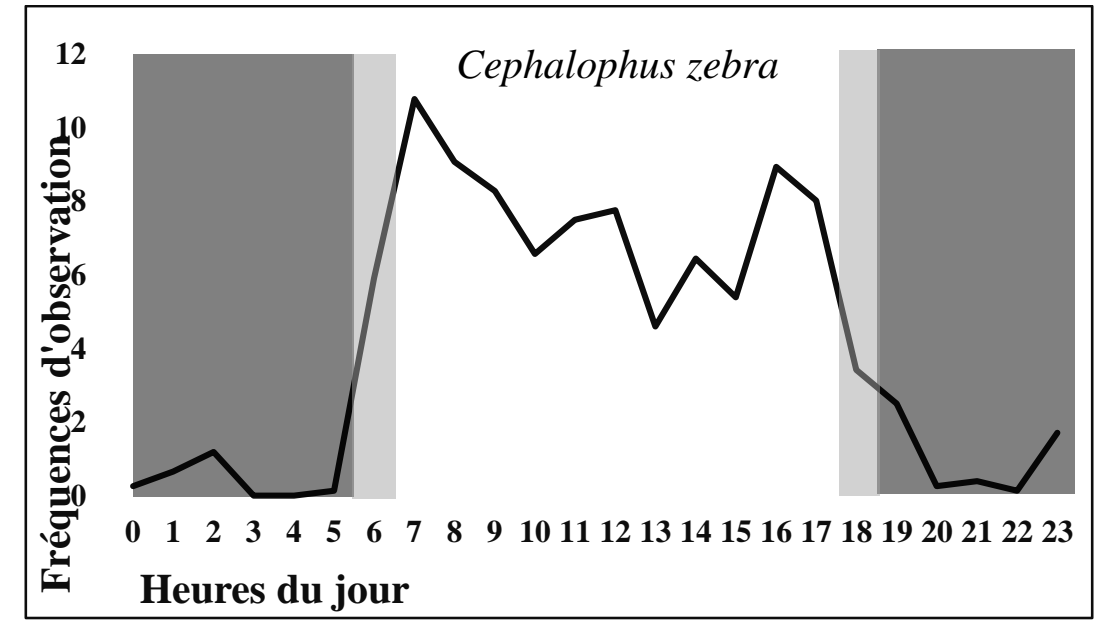

Noir $=$ Nuit $\quad$ Gris $=$ Crépuscule $\quad$ Blanc $=$ Journée

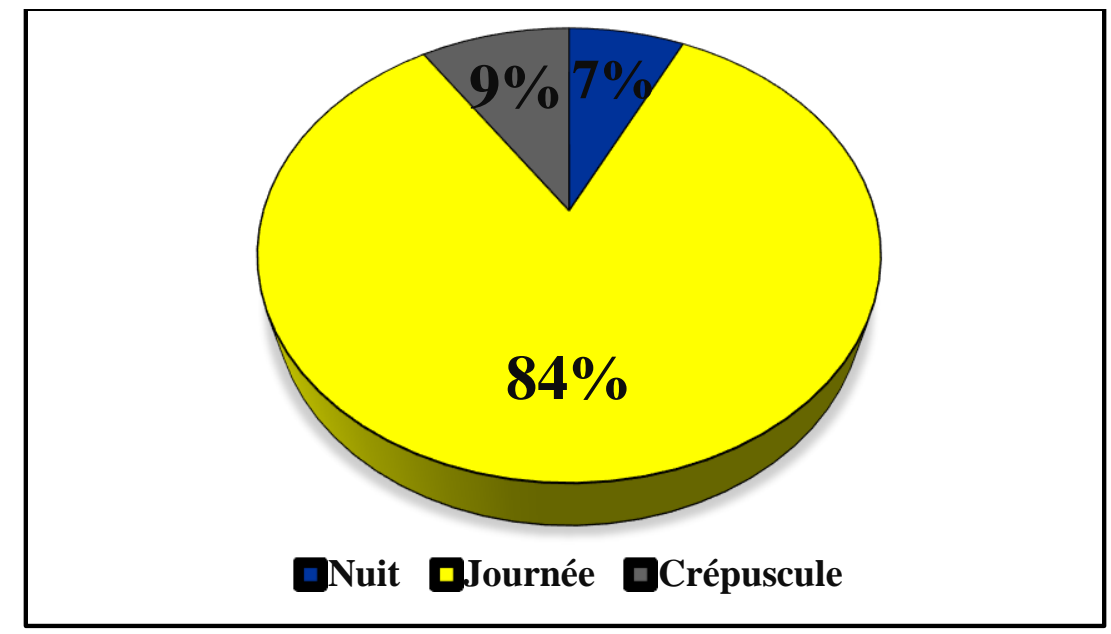

Cephalophus zebra

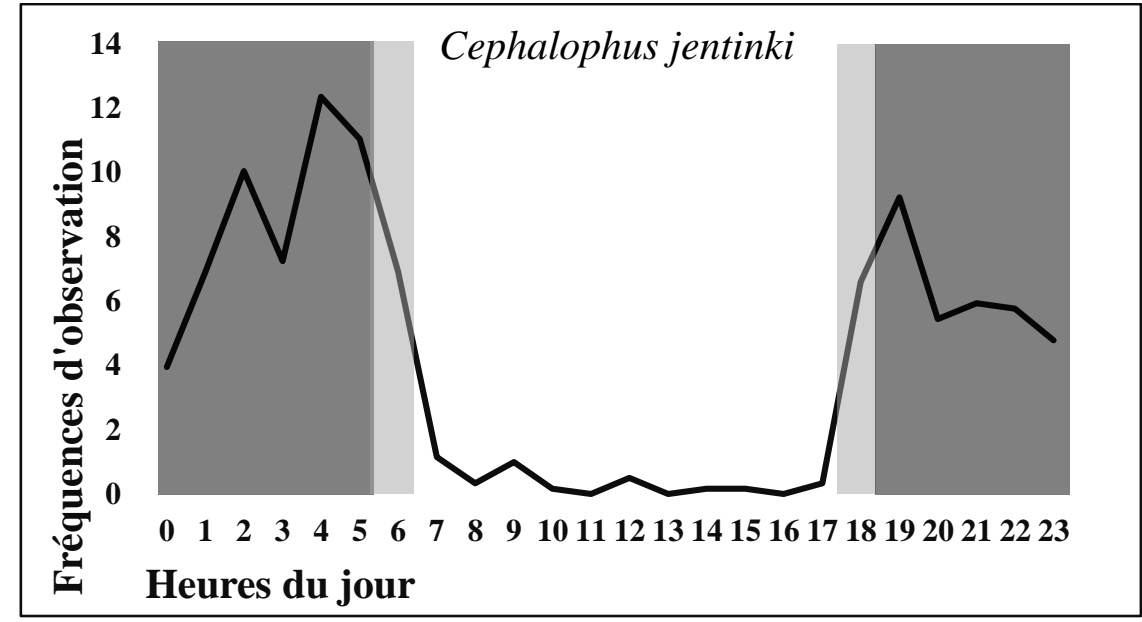

Noir $=$ Nuit $\quad$ Gris $=$ Crépuscule $\quad$ Blanc $=$ Journée

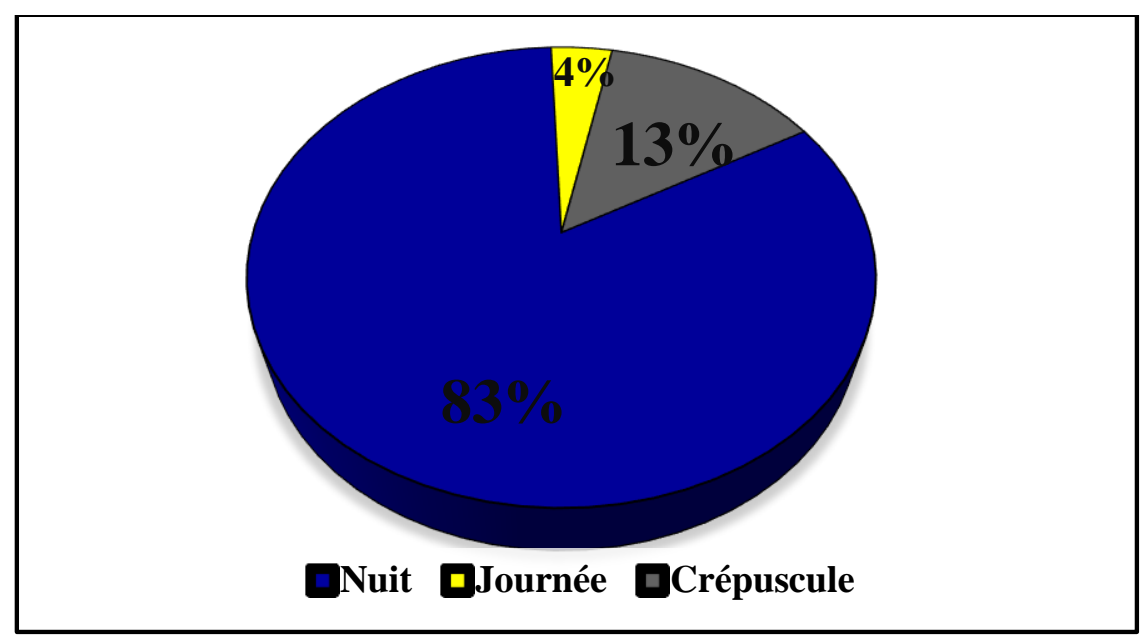

Cephalophus jentinki

Figure 2 : Fréquence d'observation de Cephalophus zebra et de Cephalophus jentinki au PNT. 
A. E. H. MONKET et al. / Int. J. Biol. Chem. Sci. 15(5): 1863-1874, 2021
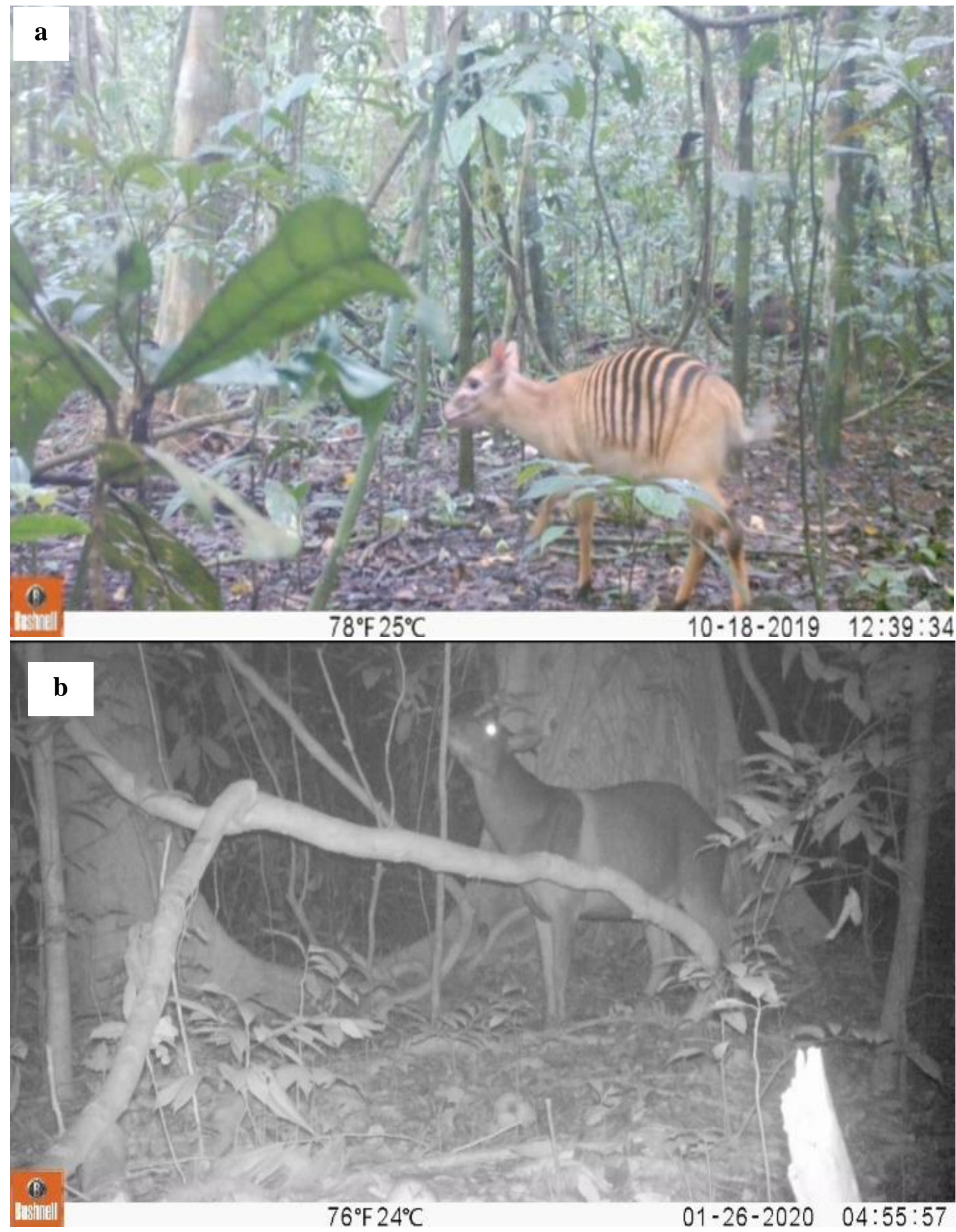

Figure 3 : Images des deux céphalophes au Parc National de Taï a : Cephalophus zebra et b : Cephalophus jentinki. 


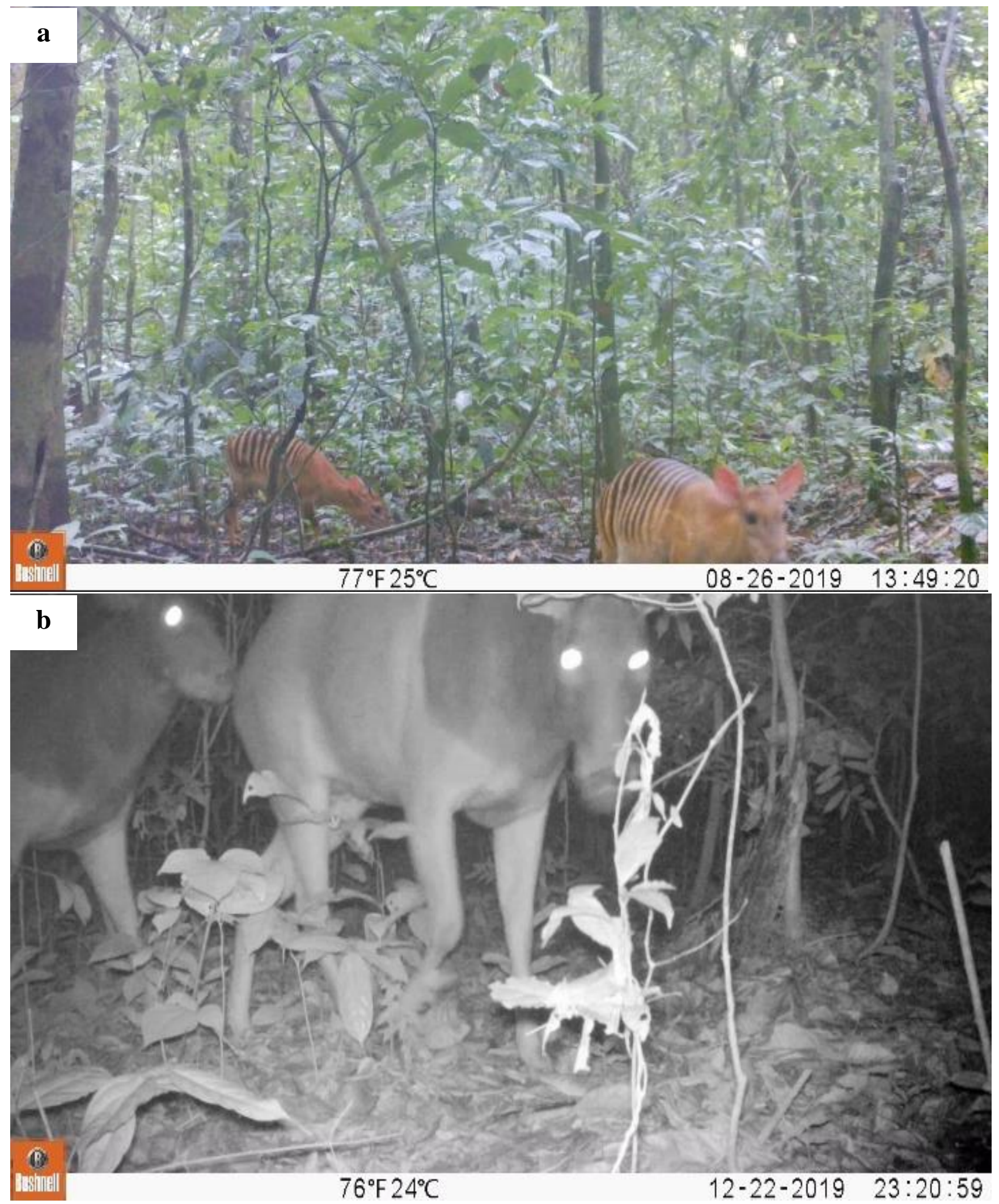

Figure 4 : Photographies de deux couples de céphalophes au Parc National de Taï a : céphalophe zébré et b : céphalophe de Jentink. 


\section{DISCUSSION}

Les deux espèces de céphalophes du Parc National de Taï concernées par cette étude ont des périodes d'activités différentes. Le céphalophe zébré a une activité principalement diurne tandis que le céphalophe de Jentink est généralement nocturne. Nos résultats concordent, d'une part, et diffèrent, d'autre part, de ceux obtenus par Newing (2001), au Libéria. Selon cet auteur, le céphalophe zébré est bien diurne que nocturne alors que le céphalophe de Jentink présente une activité cathémérale. Cette différence au niveau de l'activité nycthémérale de céphalophe de Jentink pourrait être liée à la méthodologie de transects linéaires appliquée par cet auteur. En effet, au cours des inventaires fauniques sur les transects linéaires, certains individus de céphalophe de Jentink observés dans la journée semblent être débusqués de leur lieu de repos par les équipes de collecte de données (Tiedoué et al., 2018).

Nos résultats sont similaires aux analyses de données de N'Goran et al. (2020) collectées dans le Parc National de Taï, à l'aide de pièges photographiques. Les études de ces auteurs avancent que le céphalophe zébré et le céphalophe de Jentink ont des activités nycthémérales respectivement à prédominance diurne et nocturne. Cette opposition des périodes d'activités entre ces deux espèces de céphalophes pourrait réduire non seulement la compétition directe sur les parcelles de nourriture mais aussi la compétition indirecte, en permettant l'exploitation de différentes ressources (Jiménez et al., 2010). En outre, elle pourrait également avoir des implications en ce qui concerne la distribution des deux espèces au PNT, car une activité diurne comme c'est le cas pour le céphalophe zébré, impliquerait une forte influence des activités anthropiques sur leur distribution (Diarrassouba et al., 2019).

En ce qui concerne la structure sociale du céphalophe zébré et du céphalophe de Jentink au Parc National de Taï, les observations d'individus solitaires se sont révélées les plus fréquentes. Une structure sociale similaire a déjà été observée chez d'autres espèces de céphalophes comme le céphalophe à dos jaune, le céphalophe noir et le céphalophe d'Ogilby au Libéria (Newing, 2001). Cette étude met en évidence le caractère généralement solitaire des céphalophes. En effet, les céphalophes ont développé un mode de défense passif contre les prédateurs (immobilité, camouflage), il serait évidemment avantageux de vivre seul plutôt qu'en troupeaux. Un animal isolé peut aisément fuir et se cacher dans la végétation, ce qui serait difficile pour un groupe (Groves et al., 2011). Par ailleurs, le mode de vie solitaire des céphalophes pourrait être lié à des stratégies de recherche de nourriture. Selon Kingdon et Hoffmann (2013), les céphalophes sont des espèces animales sédentaires et territoriales qui vivent sur de petits territoires qu'ils défendent par des sécrétions d'hormones. Aussi, ils ont un régime alimentaire composé principalement de fruits (jusqu'à $90 \%$ de leur régime) et de feuilles, la vie solitaire pourrait être donc une stratégie pour réduire les concurrences sur les ressources alimentaires (Jiménez et al., 2010). Ainsi, nous pouvons dire que les groupes de céphalophe zébré et de céphalophe de Jentink, constitués de deux individus adultes, seraient des formations de couples pour les périodes de rut (Groves et al., 2011). Selon les études menées sur les bovidés au Zimbabwe par ces auteurs, chez les bovidés solitaires, les individus vivent isolés dans un domaine vital bien défini pendant une grande partie de l'année. La distance critique individuelle reste grande sauf au moment du rut ou mâles et femelles se réunissent pour la reproduction (Groves et al., 2011). Le couple qui se forme est éphémère et limité à la courte période de rut. En somme, leur mœurs solitaire ou de couple semble avoir un avantage sélectif dans la reproduction et pourrait conférer à ces différentes espèces de céphalophes une certaine résistance par la conservation de gènes sauvages (rudimentaires) en minimisant le phénomène des effets de consanguinité (Estes, 1991 ; Castello, 2016).

L'immobilité de jeunes individus de céphalophes au cours de cette étude, peut-être expliquée par le fait que les céphalophes garderaient leurs petits dans les tanières. En effet, les céphalophes ont adopté ce comportement dans le but de protéger les petits contre toute agression extérieure (Kingdon et Hohhmann, 2013).

\section{Conclusion}

Cette étude par pièges photographiques menée au Parc National de Taï a permis d'établir simultanément, les périodes d'activités nycthémérales et la structure sociale du céphalophe zébré et du céphalophe de Jentink. Le céphalophe zébré a une activité préférentiellement diurne dont $84 \%$ des activités ont été faits dans la journée contre $9 \%$ au crépuscule et $7 \%$ la nuit. Cette activité diurne présente des heures d'activités maximales en début de journée (de 6 à $8 \mathrm{~h}$ ) et en fin d'après-midi (de 15 à 17h). Par contre, le céphalophe de Jentink est principalement nocturne avec $83 \%$ des activités observées la nuit contre $13 \%$ activités crépusculaires et $4 \%$ des activités de la journée. Cette espèce a été vivement active autour de $2 \mathrm{~h}$, de $4 \mathrm{~h}$ et $19 \mathrm{~h}$. Concernant le mode de vie, ces deux espèces de céphalophes sont généralement solitaires dans ce 
parc. En effet, pour le céphalophe zébré, 94,21\% des individus vivent seul contre $5,79 \%$ en groupes. Pour le céphalophe de Jentink, 96,70\% des individus solitaires contre $3,30 \%$ en groupes ont été observés. Ces espèces animales forment parfois de petits groupes constitués de deux individus adultes qui pourraient-être des couples. La présente étude fournit des informations complémentaires sur ces deux céphalophes (Cephalophus zebra et Cephalophus jentinki) présents dans le PNT et établit une base de référence pour la recherche et le développement d'une gestion efficace de la faune dans cet écosystème de forêt tropicale.

\section{CONFLIT D'INTERETS}

Les auteurs déclarent qu'il n'y a aucun conflit d'intérêts pour cet article.

\section{CONTRIBUTIONS DES AUTEURS}

AEHM a collecté les données. AEHM, CYK et MRK ont fait le traitement statistique et la rédaction du manuscrit. Tous les autres co-auteurs ont contribué à la relecture de versions antérieures de cet article.

\section{REFERENCES}

Ancrenaz M, Andrew JH, Ross J, Sollmann R, Wilting A. 2012. Handbook for wildlife monitoring using camera traps. BBEC II Secretariat publication, Kota Kinabalu, Malaisie, 57p.

Bezerra BM, Bastos M, Souto A, Keasey MP, Eason P, Schiel N, Jones G. 2014. Camera trap observations of nonhabituated critically endangered wild blonde capuchins, Sapajus flavius (formerly Cebus flavius). International Journal of Primatology, 35 : 865-907.

Brou YT. 2009. Impacts des modifications bioclimatiques et de l'amenuisement des terres forestières dans les paysanneries ivoiriennes : quelles solutions pour une agriculture durable en Côte d'Ivoire. Cuadernos Geographicos, 45 : 13-29.

Castello JR. 2016. Bovids of the World. Antelops, gazelles, cattle, goats, sheep and relatives. Princeton University Press: Princeton, NJ, USA; Oxford, UK.

Diarrassouba A, Gnagbo A, Kouakou CY, Campbell G, Tiedoué MR, Tondossama A, Kühl HS, Koné I. 2019. Differential response of seven duiker species to human activities in Taï National Park, Côte d'Ivoire. African Journal of Ecology, 1-11.

Estes RD. 1991. The behavior guide to African Mammals Including hoofed mammals, carnivores, Primates. Drawing by Daniel Otte: Foreword by E.O. Wilson, 611 p.

Gaynor KM, Branco PS, Long RA, Gonçalves DD, Granli PK \& Poole JH. 2018. Effets of human settlement and roads on diel activity patterns of elephants (Loxodonta africana). African Journal of Ecology, 56: 872-881.

Gómez H, Wallace RB, Ayala G, Tejada R. 2005. Dry season activity periods of some Amazonian mammals. Studies on Neotropical Fauna and Environment, 40: 91-95. DOI: https://doi.org/10.1080/01650520500129638

Green SE, Davidson Z, Kaaria T, Doncaster CP. 2018. Do wildlife corridors link or extend habitat? Insights from elephant use of a Kenyan wildlife corridor. African Journal of Ecology, 56: $860-871$.

Groves CP, Grubb P, Wilson DE \& Mittermeier RA. 2011. Family Bovidae (hollow-horned ruminants). In Hanbook of the World (Vol.2. Hoofed Mammals), Wilson DE, Mittermeier RA. Lynx Edicions: Barcelona, Spain; 755-773.

Hedwig D, Kienast I, Bonnet M, Curran BK, Courage A, Boesch C, Kühl HS, King T. 2018. A camera trap assessment of the forest mammal community within the transitional savannahforest mosaic of the Batéké Plateau National Park, Gabon. African Journal of Ecology, 56: 777-790.

Howe EJ, Buckland ST, Després-Einspenner ML, Kühl HS. 2017. Distance sampling with camera traps. Methods in Ecology and Evolution, 8: 1558-1565. DOI: https://doi.org/10.1111/204110X.12790

Jiménez CF, Quintana H, Pacheco V, Melton D, Tello G. 2010. Camera trap survey of medium and large mammals in a montane rainforest of northern Peru. Revista Peruana de Biologia, 17: 191-196. DOI : https://doi/org/10.15381/rpb/v17i2.27

Kely MR. 2020. Distribution, abondance, structure sociale et activité de l'éléphant de forêt (Loxodonta africana cyclotis Matschie, 1900) au Parc National de Taï (sud-ouest de la Côte d'Ivoire). Thèse de Doctorat, UFR Environnement, Université Jean Lorougnon Guédé, Côte d'Ivoire, $134 \mathrm{p}$

Kingdon J, Happold D, Butynski T, Hoffmann M, Happold M, Kalina J. 2013. Mammals of Africa (Vol. 1-6) Bloomsbury Publishing: London.

Kingdon J, Hoffmann M. 2013. Mammals of Africa. (Volume V1: Pigs, hippopotamuses, chevrotain, giraffes, deer and bovids). Bloomsbury Publishing: London. 
Kuper WJH, Sommer JC, Lovett J, Mutke HP, Linder HJ, Beentje S, Van Rompaey C, Chatelain M, Sosef W, Barthlott. 2004. Africa's hotspots of biodiversity redefined. Annals of the Missouri Botanical Garden, 91: 525-535.

Koné MS, Konate K, Yéo KP, Kouassi KE. 2014. Effects of management intensity on ant diversity in cocoa plantation (Oume, Centre west Côte d'Ivoire). Journal of Insect Conservation, 18 : 701-712.

Lauginie F. 2007. Conservation de la nature et aires protégées en Côte d'Ivoire. CEDA/NEI, Abidjan, Côte d'Ivoire. 668p.

McCarthy MS, Després-Einspenner M-L, Samuni L, Mundry R, Lemoine S, Preis A, Wittig RM, Boesch C, Kühl HS. 2018. An assessment of the efficacy of camera traps for studying demographic composition and variation in chimpanzees (Pan troglodytes). American Journal of Primatology, 80 : 22904.

Mittermeier RAWR, Turner FW, Larsen TM, Brooks C, Gascon. 2011. Global Biodiversity Conservation: The critical role of hotspots. In Biodiversity Hotspots: Distribution and Protection of Conservation Priority Areas, Zachos FE, Habel JC (Eds). Springer: Berlin Heidelberg; 3-22.

Myers NRA, Mittermeier CG, Mittermeier GAB, da Fonseca, Kent J. 2000. Biodiversity hotspots for conservation priorities. Nature, 403 : 853-858.

Newing H. 2001. Bushmeat hunting and management: Implications of duikers ecology and interspecific competition. Biodiversity and Conservation, 10: 99-118.

N'Goran NSP, Cappelle N, Bitty EA, Normand E, Kablan YA. 2020. Détermination par caméra piège des périodes d'activité de quelques mammifères terrestres au Parc National de Taï. International Journal of Biological and Chemical Sciences, 14(5): 1673-1688. DOI : https://doi.org/10.4314/ijbcs.v14i5.15

Ross J, Hearn AJ, Johnson PJ, Macdonald DW. 2013. Activity patterns and temporal avoidance by prey in response to Sunda clouded leopard predation risk. Journal of Zoology, 290: 96-106. DOI : https://doi.org/10.1111/jzo.12018.

Tiedoué MR, Normand E, Diarrassouba A, Tondossama A, Boesch C. 2015. Etat de conservation du Parc national de Taï : Résultats du suivi écologique, Phase 10. Office Ivoirien des Parcs et Réserves/Direction de Zone SudOuest. Soubré, Côte d'Ivoire, 38p.

Tiedoué MR, Diarrassouba A, Tondossama A. 2016. Etat de conservation du Parc national de Taï : Résultats du suivi écologique, Phase 11. Office Ivoirien des Parcs et Réserves/Direction de Zone Sud-Ouest. Soubré, Côte d'Ivoire, 31p.

Tiedoué MR, Koné SS, Diarrassouba A, Tondossama A. 2018. Etat de conservation du Parc national de Taï : Résultats du suivi écologique, Phase 12. Office Ivoirien des Parcs et Réserves/Direction de Zone Sud-Ouest. Soubré, Côte d'Ivoire, 37p.

Yéo KS, Konate S, Tiho, Camara KS. 2011. Impacts of land use types on ant communities in a tropical forest margin (Oume Côte d'Ivoire). African Journal of Agricultural Research, 6: 260-274.

Wearn OR, Glover-Kapfer P. 2017. Camera-trapping for Conservation: a Guide to Best-practices. WWF Conservation Technology, Series 1(1): United Kingdom; 180 p. 